\title{
Additive-Manufacturing-Enabled Air-Filled Substrate Integrated Waveguide Microwave Components
}

\author{
Kamil Yavuz Kapusuz, Sam Lemey, Piet Demeester and Hendrik Rogier \\ Department of Information Technology, Ghent University/IMEC, \\ Technologiepark-Zwijnaarde 15, 9052 Gent, Belgium \\ KamilYavuz.Kapusuz@UGent.be
}

\begin{abstract}
A 3D-printed air-filled substrate integrated waveguide topology is proposed to mitigate the problems limiting the performance of dielectric-filled substrate integrated waveguide based components while maintaining its most interesting features. The proposed topology is showcased through the design of a 3D-printed coaxial-to-AFSIW transition and a power divider/combiner. Measurement results validate the excellent performance in terms of impedance matching and bandwidth.
\end{abstract}

\section{INTRODUCTION}

In recent years, the advent of the Internet of Things (IoT) requires a novel class of cost-effective, high-performance wireless systems that facilitate invisible integration into everyday objects without significantly modifying their size, weight, aesthetics or original purpose [1]. Besides, such systems must support high data rates as required by the next generation wireless communication [1]. Therefore, researchers from both academia and industry are concentrating massive efforts on the development of novel implementation techniques that yield high performance at low manufacturing cost by exploiting lowcost materials and by adopting novel, innovative manufacturing processes to attain high accuracy based on fast prototyping.

Several innovative materials and various manufacturing techniques have been proposed to realize wireless systems for the IoT [2]. Among them, additive manufacturing, and especially $3 \mathrm{D}$ printing, is commonly preferred because of its low implementation cost and prototyping time. Nonetheless, considering the importance of the material's extrusion speed and temperature, layer thickness, infill pattern and percentage, a proper combination of this large number of options needs to be carefully controlled to achieve the desired dielectric properties and to obtain a reproducible manufacturing process for dielectric-filled microwave structures [3]. To mitigate the effect of this large set of fabrication settings on performance and to reduce dielectric losses of such dielectric-filled 3D printed structures, a novel cost effective and high performance air-filled substrate integrated waveguide (AFSIW) solution for the IoT applications was proposed in [4].

In this contribution, we describe a manufacturingindependent AFSIW topology to produce passive microwave components for smart surface applications [4]. Specially, we discuss some novel microwave components based on this AFSIW technology, which can be part of a new generation

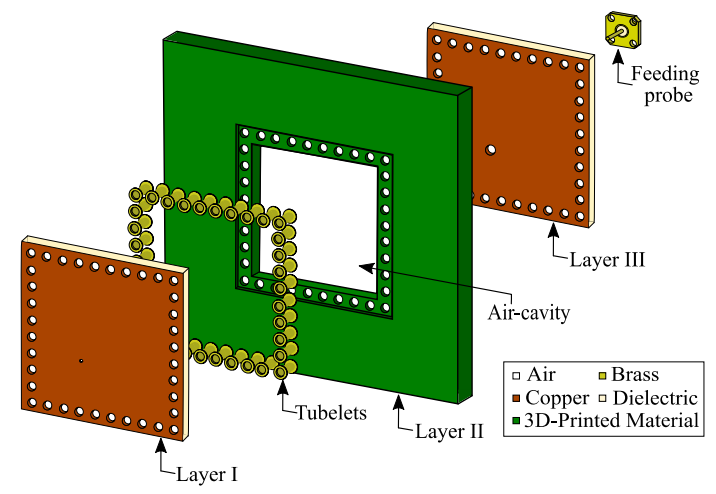

Fig. 1. Architecture of the 3D printed-material-based AFSIW technology. of cost-effective high-performance smart surface microwave systems. All the designed components were optimized for operation in the [5.15 - 5.85] $\mathrm{GHz}$ unlicensed national information infrastructure (U-NII) radio bands.

\section{AIR-FILLED SIW}

An improved AFSIW architecture [Fig. 1] was proposed in [4], enabling cost-effective realization of high-performance microwave components by inserting air-filled regions at welldefined locations and by deploying conductive tubelets to confine the electromagnetic fields within those carefully designed regions. It makes use of air as a dielectric substrate to reduce losses, and metal tubelets as conductive sidewalls. This makes the microwave components properties independent of the lossy material's characteristics in which the structure is implemented. It also reduces cost and while maintaining good performance for passive microwave component design.

The AFSIW topology consists of two conductive layers forming the upper and lower conductor walls, one layer of 3D printed material to fix the waveguide height and lowcost conductive tubelets realizing the conductive sidewalls. A well defined air cavity in the 3D printed layer acts as principal transmission medium for the electromagnetic fields. To avoid that electromagnetic fields penetrate the lossy 3D printed layer, sidewalls are implemented by closely-spaced via holes near the cavity's edges. There are realized by punching conductive tubelets through the holes, thereby joining all layers. By judiciously patterning these low-cost brass tubelet sidewalls and the air cavity, the functionality of the microwave component is defined. 


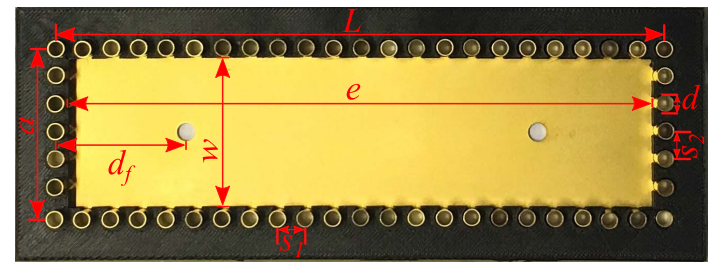

(a)

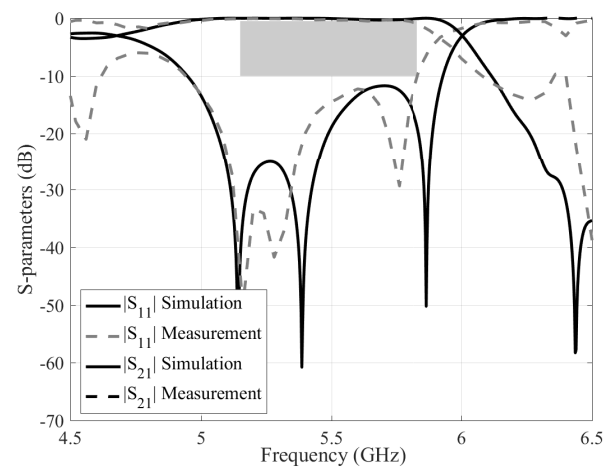

(b)

Fig. 2. Straight waveguide. (a) 3D printed material aligned with bottom conductor layer. (b) Scattering parameters. Optimized dimensions: $a=40.3 \mathrm{~mm}, w=36.27 \mathrm{~mm}, L=143.0 \mathrm{~mm}, e=138.0 \mathrm{~mm}, d_{f}=30.51 \mathrm{~mm}$, $s_{1}=s_{2}=6.5 \mathrm{~mm}$, and $d=4.0 \mathrm{~mm}$. The thickness of the 3D printed material is $2.78 \mathrm{~mm}$.

\section{Additive Manufactured Components}

\section{A. 3D-Printed AFSIW with Coaxial-to-AFSIW Transition}

First, a $143 \mathrm{~mm}$-long straight waveguide with short-ended coaxial-to-air-filled SIW transition [Fig. 2(a)] is designed. For this component, the design procedure described in [4] was adopted to obtain negligible loss and substrate-independent behaviour. Fig. 2(b) shows the simulated and measured scattering parameters of the realized prototype. Its measured insertion loss only varies between $0.23 \mathrm{~dB}$ and $0.65 \mathrm{~dB}$, in the complete $5.15 \mathrm{GHz}$ to $5.85 \mathrm{GHz}$ frequency band.

\section{B. Power Divider/Combiner}

The geometry of the designed power divider/combiner based on AFSIW architecture is depicted in Fig. 3. Eight inductive posts are applied to optimize return and insertion loss. As seen in Fig. 3. a), there are no dielectric slabs required for the realization of these inductive posts, in contrast to [5]. Moreover, additional tubelets may be implemented near the AFSIW sidewalls to further enhance mechanical stability, as shown in Fig. 3. a). The simulated and measured performances are illustrated in Fig. 3 (b), where a very good agreement between simulation and measurement results is observed.

\section{CONCLUSiON}

The design and implementation of an AFSIW straight waveguide and four-way power divider with coaxial-to-airfilled SIW transition are presented to achieve good input matching, low insertion loss and high isolation. The proposed 3D-printed AFSIW technology enables designers to implement arbitrarily shaped planar SIW structures with arbitrary thickness in a cost-effective manner, while avoiding

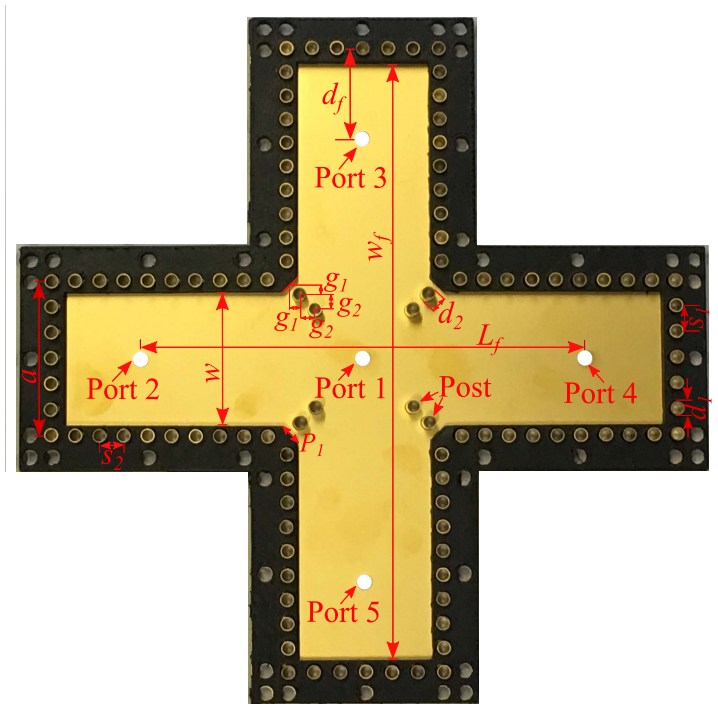

(a)

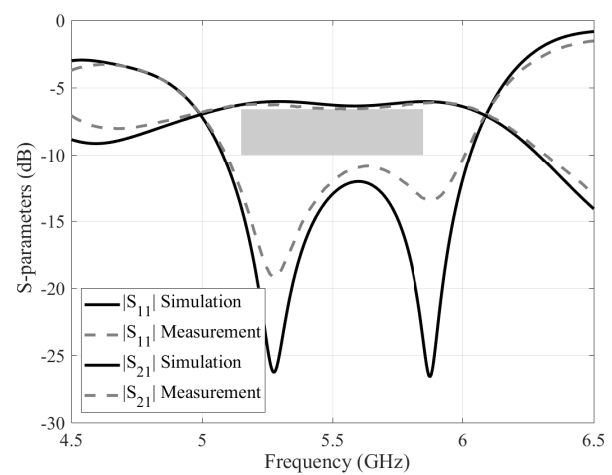

(b)

Fig. 3. Power divider/combiner. (a) 3D printed material aligned with bottom conductor layer. (b) Scattering parameters. Optimized dimensions: $w=39.13 \mathrm{~mm}, a=45.0 \mathrm{~mm}, L_{f}=130.6 \mathrm{~mm}, w_{f}=175.5 \mathrm{~mm}$, $d_{f}=25.7 \mathrm{~mm}, s_{1}=7.0 \mathrm{~mm}, s_{2}=7.5 \mathrm{~mm}, p_{1}=5.66 \mathrm{~mm}, d_{1}=d_{2}=4.0 \mathrm{~mm}$, $g_{1}=2.75 \mathrm{~mm}$, and $g_{2}=4.6 \mathrm{~mm}$. The thickness of the 3D printed material is $3.15 \mathrm{~mm}$.

its disadvantages, being a performance that strongly depends on material and process parameters. Therefore, well-defined air-filled regions are inserted into the 3D printed material. Moreover, the electromagnetic fields are confined through SIW sidewalls, formed by low-cost tubelets that also join the different layers of the stack-up.

\section{REFERENCES}

[1] S. Lemey et. al, "Threefold rotationally symmetric SIW antenna array for ultra-short-range MIMO communication," IEEE Trans. Antennas Propag., vol. 64, no. 5, pp. 1689-699, May 2016.

[2] M. Bozzi et. al, "Novel materials and fabrication technologies for SIW components for the Internet of Things," in IEEE Int. Workshop on Electromagn.: Appl. and Student Innovation Competition (iWEM), Nanjing, China, 2016, pp. 1-3.

[3] E. Massoni et. al, "3-D printed substrate integrated slab waveguide for single-mode bandwidth enhancement," IEEE Microwave and Wireless Components Letters, vol. 27, no. 6, pp. 536-538, June 2017.

[4] K.Y. Kapusuz, S. Lemey, and H. Rogier, "Substrate independent microwave components in substrate integrated waveguide technology for high-performance smart surfaces," IEEE Trans. Microw. Theory Techn., Accepted for publication.

[5] F. Parment et. al,"Air-filled substrate integrated waveguide for low-loss and high power-handling millimeter-wave substrate integrated circuits," IEEE Trans. Microw. Theory Techn., vol. 63, no. 4, pp. 1228-1238, Apr. 2015. 